\title{
Transforming guided waves with metamaterial waveguide cores
}

\author{
S. Viaene*a,b, V. Ginis ${ }^{\mathrm{a}}$, J. Danckaert ${ }^{\mathrm{a}}$, P. Tassin ${ }^{\mathrm{b}, \mathrm{a}}$ \\ ${ }^{a}$ Applied Physics Research Group, Vrije Universiteit Brussel, Pleinlaan 2, B-1050, Brussel, \\ Belgium; \\ ${ }^{b}$ Department of Physics, Chalmers University of Technology, SE-412 96, Göteborg, Sweden.
}

\begin{abstract}
Metamaterials make use of subwavelength building blocks to enhance our control on the propagation of light. To determine the required material properties for a given functionality, i.e., a set of desired light flows inside a metamaterial device, metamaterial designs often rely on a geometrical design tool known as transformation optics. In recent years, applications in integrated photonics motivated several research groups to develop two-dimensional versions of transformation optics capable of routing surface waves along graphene-dielectric and metal-dielectric interfaces. Although guided electromagnetic waves are highly relevant to applications in integrated optics, no consistent transformation-optical framework has so far been developed for slab waveguides. Indeed, the conventional application of transformation optics to dielectric slab waveguides leads to bulky three-dimensional devices with metamaterial implementations both inside and outside of the waveguide's core. In this contribution, we develop a transformationoptical framework that still results in thin metamaterial waveguide devices consisting of a nonmagnetic metamaterial core of varying thickness [Phys. Rev. B 93.8, 085429 (2016)]. We numerically demonstrate the effectiveness and versatility of our equivalence relations with three crucial functionalities: a beam bender, a beam splitter and a conformal lens. Our devices perform well on a qualitative (comparison of fields) and quantitative (comparison of transmitted power) level compared to their bulky counterparts. As a result, the geometrical toolbox of transformation optics may lead to a plethora of integrated metamaterial devices to route guided waves along optical chips.
\end{abstract}

Keywords: Transformation optics, Metamaterials, Waveguide integration, Dispersion engineering, Slab waveguide.

\section{INTRODUCTION}

Geometrical ideas crucially enhance our understanding and control of light in optical devices. The design of many optical components with slowly varying material properties is based on the ray picture of light, assuming that the phase of electromagnetic waves determines its propagation [1]. Mathematically, the ray picture of light boils down to an elegant formula, put forward by Fermat. The principle of Fermat states that light rays follow those trajectories with extremal optical path lengths (OPL), proportional to the refractive index of the medium:

$$
O P L=\int d \boldsymbol{r} n(\boldsymbol{r}) .
$$

In the past century, several scientists pointed to the resemblance of the behavior of light rays in inhomogeneous media and the propagation of light through nontrivial space-times [2-4]. Indeed, in general relativity light also propagates along trajectories, known as geodesics of a space-time geometry, which extremize the path length.

With the advent of transformation optics [5-9] and metamaterials [10-12], the link between the propagation of light through inhomogeneous media and the propagation of light through curved spaces became an observable fact. The Maxwell equations on a nontrivial space $\mathrm{g}_{\mathrm{ij}}$ are exactly imposed by a specific electromagnetic metamaterial if the material's electric (permittivity $\varepsilon^{i j}$ ) and magnetic (permeability $\mu^{i j}$ ) material distributions satisfy analytical equivalence relations [5]

$$
\varepsilon^{i j}=\sqrt{g} g^{i j}=\mu^{i j} .
$$

As a result, light behaves inside a transformation-optical metamaterial as if it propagates inside the (nontrivial) space.

*sophie.viaene@vub.ac.be

Metamaterials X, edited by Allan D. Boardman, Nigel P. Johnson, Kevin F. MacDonald, Ekmel Özbay, Proc. of SPIE Vol. 9883, 98831F · C 2016 SPIE · CCC code: 0277-786X/16/\$18 - doi: 10.1117/12.2227501 
Besides the equivalence relations [Eq. (1)], the application of a coordinate transformation is a crucial ingredient of transformation optics. Coordinate transformations design the eventual light flows inside a metamaterial device. As shown in Fig. 1a-c, a coordinate transformation deforms initially straight light flows in a trivial Euclidean geometry $\delta_{i j}$ so that they follow a desired trajectory, e.g., they bend over 90 degrees. These trajectories are geodesics of the coordinate-induced geometry $g_{i j}$, which may be expressed in terms of the Jacobian $\Lambda^{i^{\prime}}{ }_{i}=\partial x^{i^{\prime}} / \partial x^{i}$ associated to the coordinate transformation from old (Euclidean) coordinates $x^{i}$ to new coordinates $x^{i}$ [3]

$$
g_{i j}=\Lambda_{i}^{i^{\prime}} \delta_{i^{\prime} j^{\prime}} \Lambda^{j^{\prime}{ }_{j}},
$$

making use of the Einstein summation convention. In conclusion, the application of a tailored coordinate transformation leads to desired light flows, following the geodesics of a nontrivial geometry $g^{i j}$, and the equivalence relations materialize them in an optical device with impedance-matched metamaterials [Eq. (1)].

The geometrical tools of transformation optics naturally extend Fermat's principle beyond the ray approximation [13]. After impressive designs of invisibility cloaks [5-9], the geometrical understanding of the interaction of light with metamaterials has not only improved our control on the flow of light [14-15], the emission of electromagnetic radiation [16-17], and our understanding of effective gauges [18-19], but also allows to study the behavior of light in space-times that are actual solutions of the Einstein equations, such as artificial black holes [20-21] and the cosmological redshift [22].

Recently, there is a growing interest to accurately manipulate light flows along two-dimensional material systems in integrated optics, such as (bio-)sensing [23-24], optical circuitry [25-26], and all-optical device actuation [27-28]. These applications benefit from strong confinement of light, which is achieved either by making use of bandgap materials [2931] or by coupling to plasmon polaritons on metal-dielectric and graphene-dielectric interfaces [32-33]. Motivated by the potential of surface wave technology, several research groups have successfully applied the existing framework of transformation optics to single metal-dielectric interfaces [34-37] and graphene-dielectric interfaces [38]. Indeed, transformation optics has no need for adaptations when it couples to metal-based surface plasmons. The energy of a surface wave, which is confined to a metal-dielectric interface, mostly resides inside the dielectric at frequencies far from the surface plasmon resonance of the metal. As a result, one may apply the conventional framework of transformation optics only to the dielectric layer to impose coordinate-based surface waves along the interface.

Unfortunately, the propagation range of surface plasmon polaritons is limited considerably by dissipation loss, especially at infrared and optical frequencies [39]. Therefore, although transformation optics may design surface flows along a metal-dielectric interface, this system is not suitable for long-range or low-loss applications in optical circuitry, actuation and sensing. To remedy the metallic losses that are inherent to metal-dielectric interfaces, researchers have turned to dielectric-dielectric (metamaterial) interfaces, making use of phonon polaritons sustained by polar dielectrics [40-41], Dyakonov surface waves along anisotropic dielectric interfaces [42] and mie-resonance based metamaterials or metasurfaces [43-45]. In recent years, the scope of all-dielectric metamaterials has systematically increased, e.g., dielectric building blocks may now sustain both positive and negative permittivity and permeability distributions, and fabrication tools such as direct laser writing with femtosecond pulses [46-47] and self-assembly [48] allow for large-area and highly anisotropic metamaterial designs.

In this contribution, we present our recent work on the manipulation of guided electromagnetic modes with low-loss alldielectric metamaterial waveguides based on a radically different formulation of transformation optics [49]. In Sec. 2, we apply a two-dimensional coordinate transformation to the symmetry plane of a dielectric slab waveguide, consisting of a high-index core layer $\varepsilon_{\text {core }}$ and low-index outer layers $\varepsilon_{\text {out }}$ such as vacuum, to mold the flow of guided modes along its surface. We notice that the straightforward application of transformation optics leads to bulky and impractical designs, with metamaterial implementation both inside and outside of the core region. To avoid bulky designs, we derive specific equivalence relations for guided electromagnetic modes, based on the effect of a two-dimensional coordinate transformation on their characteristic equations. Our equivalence relations lead to feasible and low loss metamaterial waveguide cores of varying thickness without need of metamaterial implementations in the outer layers. In Sec. 3, we demonstrate the flexibility and effectiveness of our equivalence relations with numerical simulations of three proof-ofprinciple devices: a beam bender, a beam splitter and a conformal lens. 
(a)

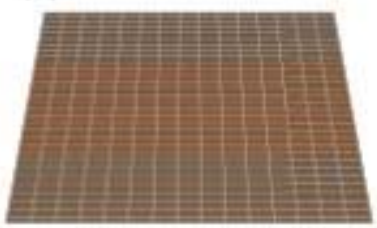

$(u, v)$

(d)

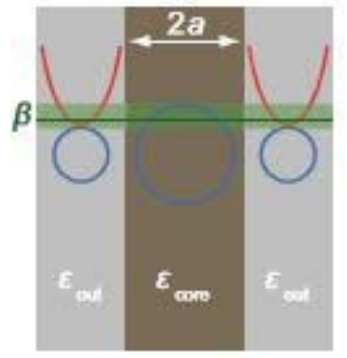

(g)

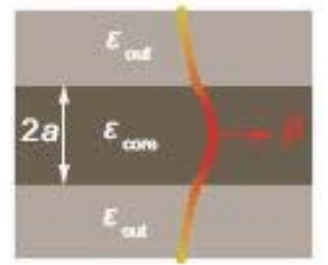

(b)

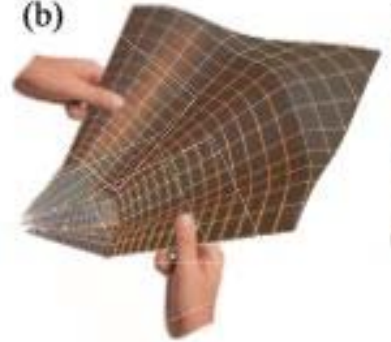

(c)

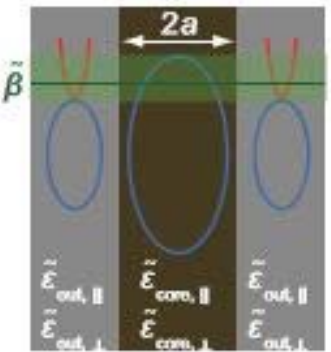

(h)

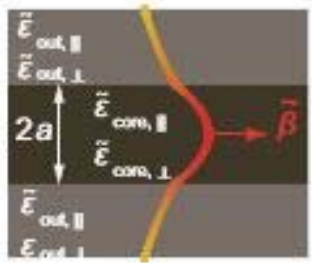

(c)

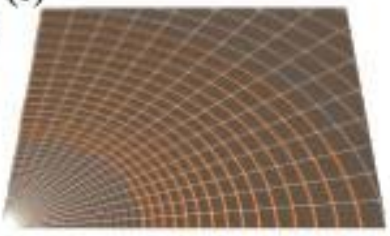

$(x, y)$

(f)

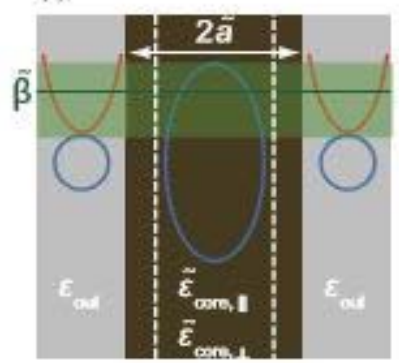

(i)

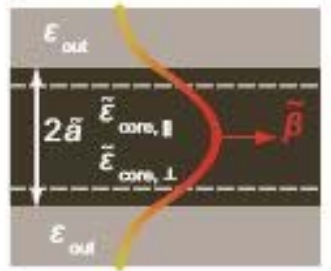

Figure 1. Our design uses a nonmagnetic uniaxial metamaterial waveguide of varying thickness to impose two-dimensional flows of light. The symmetry plane of a slab waveguide (a) is locally stretched by a two-dimensional conformal coordinate transformation (b) so that light bends over 90 degrees as if it experiences a geometry $\gamma(x, y)$ (c). (d) Accordingly, the vector space of the incident guided mode with propagation constant $\beta$ (green) is stretched in the symmetry plane (e). The total wave vectors lie respectively on elliptical (hyperbolic) isofrequency contours of the wave equation in blue (red) inside the core (outer) region. According to the traditional recipe of transformation optics, the exponential tails $\left(k_{l}\right)$ and thickness $a$ are preserved because metamaterials are implemented in the core and outer layers. (f) To preserve confinement and to impose a globally stretched propagation vector without metamaterials in the outer layers, a thickness variation $\tilde{a}$ ensures the continuity conditions at the interfaces at the expense of changes in the exponential tails $\left(k_{l}\right)$. $(\mathrm{g})$ The initial transversemagnetic profile (with intensity in color online) is either stretched in a global way by nontrivial materials in both the core and the outer regions $(\mathrm{h})$ or in a partial way by one nontrivial material in the core region complemented with a thickness variation (i).

\section{TWO-DIMENSIONAL TRANSFORMATION OPTICS}

As shown in Fig. 1a-c, confined light flows along the symmetry plane of a slab waveguide are manipulated by twodimensional coordinate transformations, e.g., a logarithmic map implementing a beam bend [15]. As a consequence, the conventional equivalence relations of transformation optics [Eq. (1)] generate material distributions that are independent of the transverse coordinate. Therefore, the conventional application of transformation optics leads to impedancematched metamaterial implementations that should, in principle, extend to infinity outside of the core layer, resulting in bulky and impractical designs. In this section, we analyze the effect of a two-dimensional coordinate transformation on the characteristic equations of a transverse-magnetic (TM) guided mode to come up with alternative and efficient equivalence relations, based upon the description of the guided mode in the reciprocal space (Fig 1d-f) and the real space (Fig. 1g-i) [29].

An incident transverse-magnetic guided mode of angular frequency $\omega$ (Fig 1d, 1g) consists of a confined transverse profile that propagates along the symmetry plane with propagation constant $\beta$. The transverse profile is confined to the 
core layer, consisting of exponentially decaying tails outside of the core characterized by an extinction coefficient $k_{I}$ and sinusoidal variations inside the core characterized by a standing wave number $k_{2}$ (Fig. $1 \mathrm{~g}$ ). The characteristic equations of a guided mode now fix the frequency-dependence of the wavevector components. First, the Helmholtz equation of a guided TM-mode relates the wavevector components, i.e., the transverse components $\left(k_{1}, k_{2}\right)$ and the parallel component $(\beta)$, at a particular frequency $\omega$ :

$$
\left[\Delta_{x y} \pm k_{1,2}^{2}+\varepsilon_{\text {core, layer }} \omega^{2} / c^{2}\right] H_{\|}=0 .
$$

In particular, it imposes circular isofrequency contours inside the core and hyperbolic isofrequency contours outside of the core (Fig. 1d). These contours are compatible for a range of propagation constants, within the green band. Second, only one particular propagation constant $\beta(\omega)$, indicated by the green line in Fig. 1d, will sustain a continuous transverse profile at the interfaces. The continuous profile on a waveguide of thickness $2 a$ is imposed by the dispersion relation of the TM-mode :

$$
\tan \left(k_{2}(\omega, \beta) a\right)=\frac{\varepsilon_{\text {core }} k_{1}(\omega, \beta)}{\varepsilon_{\text {out }} k_{2}(\omega, \beta)} .
$$

We now investigate how the Helmholtz equation and the dispersion equation change when a two-dimensional conformal transformation is applied to the symmetry plane of the slab waveguide. A conformal transformation locally rescales all in-plane vectors with a stretching $X$ and rotates them without changing their relative angles [15, 50]. Equation (2) readily extracts the induced geometry, which has isotropic in-plane components $\gamma_{i j}=\gamma(x, y) \delta_{i j}$ and does not change the transverse components. As mentioned before, the conventional application of transformation optics introduces metamaterial implementations both inside and outside of the core, i.e., $\varepsilon_{\text {core }, \text { out } \perp}=\gamma(x, y) \varepsilon_{\text {core, out }}, \quad \varepsilon_{\text {core, out } \|}=\varepsilon_{\text {core out }}, \quad \mu=1$.

(Fig. 1e, 1h). This is a necessary requirement to preserve both the transverse wavevector components $k_{1}$ and $k_{2}$ while stretching the in-plane propagation $\tilde{\beta}=X \beta$. Notice that, because the entire transverse profile is exactly preserved, the dispersion relation preserves the thickness $2 a$ of the metamaterial waveguide.

In Ref. [49], we start from the characteristic equations of the guided modes to derive alternative equivalence relations. To impose the in-plane propagation, we insert a metamaterial inside the core layer that reproduces the Helmholtz equation of the induced geometry, i.e., a unidirectional nonmagnetic core whose anisotropy is proportional to the induced geometry

$$
\varepsilon_{\perp}=\gamma(x, y) \varepsilon_{\text {core }}, \quad \varepsilon_{\|}=\varepsilon_{\text {core }}, \quad \mu=1
$$

As a consequence, the sinusoidal variations inside the core are preserved $\left(\tilde{k}_{2}=k_{2}\right)$. In contrast, because we explicitly do not implement a metamaterial in the outer layers, the Helmholtz equation will change the extinction coefficient $\tilde{k}_{1}^{2}=\gamma(x, y) \beta^{2}-\varepsilon_{\text {out }} \omega^{2} / c^{2}$. To make sure that the profile is continuous at the interfaces, i.e., that the transverse profile propagates with a transformed propagation constant $\tilde{\beta}=\sqrt{\gamma(x, y)} \beta \approx X \beta$ at the incident frequency $\omega$, we impose a geometry-dependent thickness variation

$$
\tilde{a}(x, y)=\frac{1}{k_{2}} \operatorname{atan}\left(\frac{\varepsilon_{\text {core }}}{\varepsilon_{\text {out }}} \frac{\sqrt{\gamma(x, y) \beta^{2}-\varepsilon_{\text {out }} \omega^{2} / c^{2}}}{k_{2}}\right) .
$$

Our alternative equivalence relations [Eqs. (5)-(6)] lead to nonmagnetic metamaterial cores of varying thickness without need for metamaterial implementations in the outer layers.

\section{NONMAGNETIC METAMATERIAL WAVEGUIDE COMPONENTS}

We will present numerical simulations of three proof-of-principle devices, a beam bender based on a logarithmic map [15], a beam splitter based on a Schwarz-Christoffel transformation [50] and a conformal lens based on a Möbius transformation [15], to demonstrate the versatility and effectiveness of our equivalence relations. Figure 2 visualizes the symmetric thickness variation (the floating surface represents the thickness variation of the upper interface) and the 
(a)

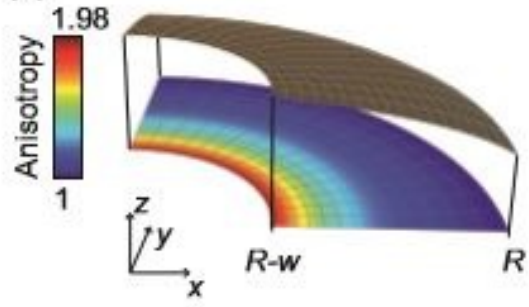

(b)

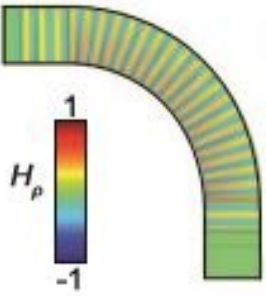

(c) (d)

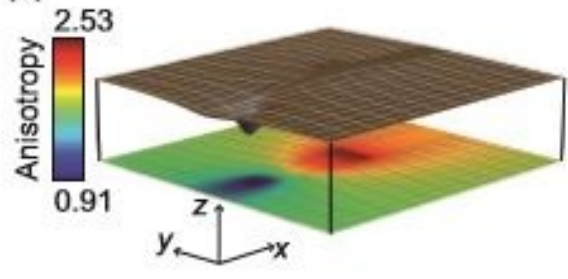

(e)

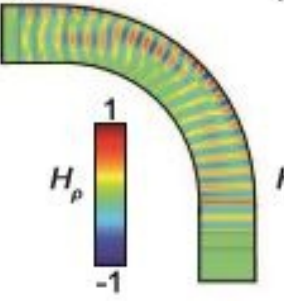

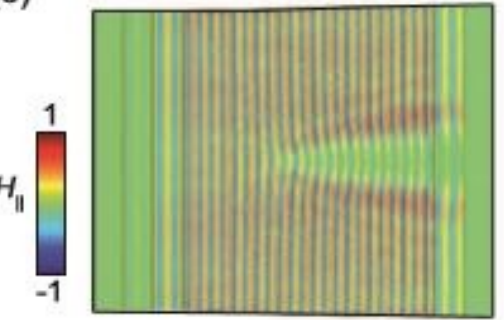

(f)

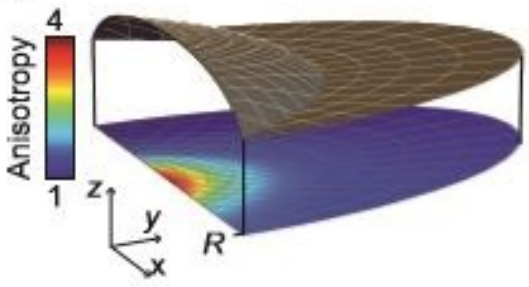

(g)

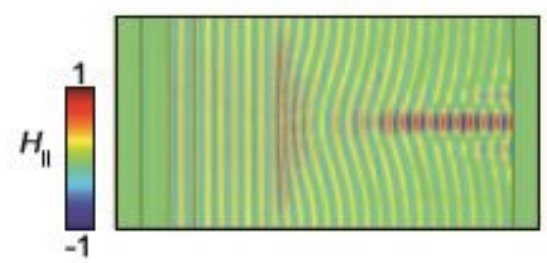

Figure 2. Demonstration and numerical verification of the versatility of the two-dimensional equivalence relations for a beam bender (a)-(c), beam splitter (d)-(e) and Möbius lens (f)-(g). Both the anisotropy (visualized by surface coloring on the symmetry plane of the waveguide) and the thickness variations of the core medium (visualized by the height of the floating surface representing the upper interface $\mathrm{z}=\tilde{\mathrm{a}}$ ) act on the in-plane magnetic fields to impose desired light flows and correspond to technologically feasible parameters. Without thickness variations (c), the in-plane magnetic fields cannot complete the bend.

anisotropy of the uniaxial metamaterial inside the core (color online on symmetry plane) for each waveguide component. The required metamaterial distributions and thickness variations are feasible and can be fabricated by 3D printing (direct laser writing) or other lithographic techniques [44-48]. Qualitatively, we observe that the in-plane magnetic fields in Figs. $2 b, 2 e$, and $2 g$, bend, split and focus in a very efficient way.

To quantitatively compare the performance of our two-dimensional designs to the bulky implementations of transformation optics, we performed several numerical simulations of beam bends with decreasing inner radius $\mathrm{R}$ while preserving the incident beam width w. As shown in Fig. 4, the throughput of our two-dimensional bends (from $84 \%$ to 93\%) is highly comparable to the three-dimensional implementations (from $86 \%$ to $95 \%$ ), even close to the subwavelength regime. In contrast, an isotropic metamaterial core supplemented by the required thickness variation cannot maintain high throughputs for small bending radii. Besides the anisotropy of metamaterial cores, the thickness variation is another crucial ingredient of the equivalence relations without which guided waves cannot complete the bend (Fig. 2c).

\section{CONCLUSION}

In this contribution, we have introduced alternative equivalence relations, based on the characteristic equations of guided modes, to design thin, nonmagnetic metamaterial waveguides of varying thickness. Next to the successful implementation of individual devices, i.e., beam benders, beam splitters and conformal lenses, our work paves the way for a holistic manipulation of guided modes: one optimized metamaterial waveguide may seamlessly split, bend and focus light in an integrated setup with minimized insertion loss [49]. 


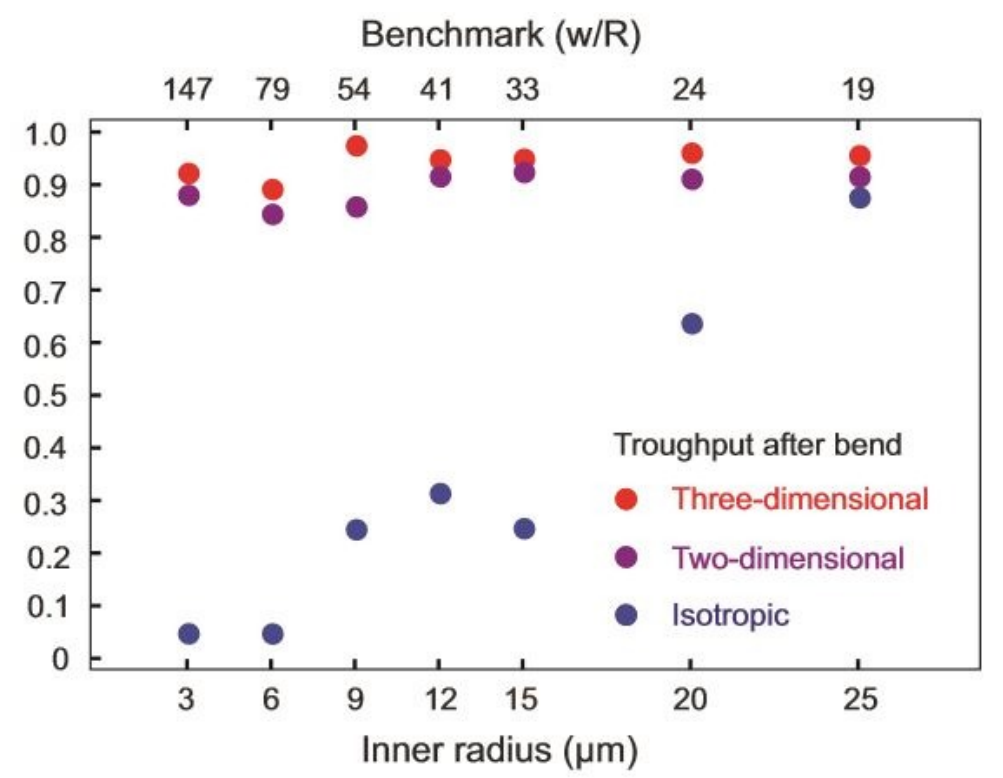

Figure 3. The throughput of our beam bender is evaluated for seven outer radii $\mathrm{R}$ at fixed beam width w. Increases in anisotropy are represented by the benchmark $\mathrm{w} / \mathrm{R}$, as a way to demonstrate the effectiveness of our equivalence relations (purple dots) compared to the conventional design of transformation optics (red dots) and an isotropic implementation with appropriate thickness variation (blue dots). The throughputs of our two-dimensional metamaterial cores are impressive, lying close to those of the three-dimensional implementation while isotropic metamaterial cores cannot maintain their performance as inner radii approach the free space wavelength $\lambda=1.5 \mu \mathrm{m}$. Mesh convergence studies resulted in negligible error bars.

\section{REFERENCES}

[1] M. Born and E. Wolf, [Principles of Optics: Electromagnetic Theory of Propagation, Interference and Diffraction of Light], Pergamon Press, New York, (1964).

[2] L.D. Landau and E.M. Lifshitz, [The classical theory of fields: Course of theoretical physics], 3rd ed., Pergamon Press, Oxford, (1971).

[3] S.M. Carroll, [Spacetime and geometry. An introduction to general relativity], Wiley, New York, (2004).

[4] F. de Felice, "On the gravitational field acting as an optical medium," General Relativity and Gravitation 2, p. 347-357 (1971).

[5] D. Schurig, J.J. Mock, B.J. Justice, S.A. Cummer, J.B. Pendry, A.F. Starr, and D.R. Smith, "Metamaterial electromagnetic cloak at microwave frequencies," Science 314, p. 977-980 (2006).

[6] U. Leonhardt, "Optical conformal mapping," Science 312, p. 1777-1780 (2006).

[7] B. Zhang, "Electrodynamics of transformation-based invisibility cloaking," Light: Science \& Applications 1, p. e32 (2012).

[8] A. Greenleaf, Y. Kurylev, M. Lassas, U. Leonhardt, and G. Uhlmann, "Cloaked electromagnetic, acoustic, and quantum amplifiers via transformation optics," Proceedings of the National Academy of Sciences 109, p. 1016910174 (2012).

[9] X. Ni, Z.J. Wong, M. Mrejen, Y. Wang, and X. Zhang, "An ultrathin invisibility skin cloak for visible light," Science 349, p. 1310-1314 (2015).

[10] J.B. Pendry, "Photonics: Metamaterials in the sunshine," Nature Materials 5, p. 599-600 (2006).

[11] N. Engheta and R. W. Ziolkowski, [Metamaterials: Physics and Engineering Explorations], Wiley, New York, (2006).

[12] C.M. Soukoulis and M. Wegener, "Past achievements and future challenges in the development of threedimensional photonic metamaterials," Nature Photonics 5, p. 523-530 (2011).

[13] J. B. Pendry, A. Aubry, D.R. Smith, and S.A. Maier, "Transformation optics and subwavelength control of light," Science 337, p. 549-552 (2012). 
[14] M. Rahm, S.A. Cummer, D. Schurig, J.B. Pendry, and D.R. Smith, "Optical design of reflectionless complex media by finite embedded coordinate transformations," Physical Review Letters 100, p. 063903 (2008).

[15] L. Xu and H. Chen, "Conformal transformation optics," Nature Photonics 9, p. 15-23 (2015).

[16] V. Ginis, J. Danckaert, I. Veretennicoff, and P. Tassin, "Controlling Cherenkov radiation with transformationoptical metamaterials," Physical Review Letters 113, p. 167402 (2014).

[17] P. Genevet, D. Wintz, A. Daniel, A. Ambrosio, A. She, R. Blanchard, and F. Capasso, "C ontrolled steering of Cherenkov surface plasmon wakes with a one-dimensional metamaterial," Nature Nanotechnology 10, p. 804809 (2015).

[18] F. Liu, and J. Li, "Gauge field optics with anisotropic media," Physical Review Letters 114, p. 103902 (2015).

[19] T. Ma, A.B. Khanikaev, S.H. Mousavi, and G. Shvets, "Guiding electromagnetic waves around sharp corners: topologically protected photonic transport in metawaveguides," Physical Review Letters 114, p. 127401 (2015).

[20] Editorial, "The power of analogies," Nature Photonics 8, p. 1 (2014).

[21] C. Sheng, H. Liu, Y. Wang, S.N. Zhu, and D.A. Genov, "Trapping light by mimicking gravitational lensing," Nature Photonics 7, p. 902-906 (2013).

[22] V. Ginis, P. Tassin, B. Craps, and I. Veretennicoff, "Frequency converter implementing an optical analogue of the cosmological redshift," Optics Express 18, p. 5350-5355 (2010).

[23] X. Fan and I.M. White, "Optofluidic microsystems for chemical and biological analysis," Nature Photonics $\mathbf{5}$, p. 591-597 (2011).

[24] A. Alu and N. Engheta, "Dielectric sensing in $\epsilon$-near-zero narrow waveguide channels," Physical Review B 78, p. $045102(2008)$.

[25] M. Law, D.J. Sirbuly, J.C. Johnson, J. Goldberger, R.J. Saykally, and P. Yang, "Nanoribbon waveguides for subwavelength photonics integration," Science 305, p. 1269-1273 (2004).

[26] Y. Yang, A.Q. Liu, L.K. Chin, X.M. Zhang, D.P. Tsai, C.L. Lin, C. Lu, G.P. Wang, and N.I. Zheludev, "Optofluidic waveguide as a transformation optics device for lightwave bending and manipulation," Nature Communications 3, p. 651 (2012).

[27] D. Van Thourhout and J. Roels, "Optomechanical device actuation through the optical gradient force," Nature Photonics 4, p. 211-217 (2010).

[28] V. Ginis, P. Tassin, C.M. Soukoulis, and I. Veretennicoff, "Enhancing optical gradient forces with metamaterials," Physical Review Letters 110, p. 057401 (2013).

[29] J.D. Joannopoulos, S.G. Johnson, J.N. Winn, and R.D. Meade, [Photonic crystals: molding the flow of light], 2nd ed., New Jersey, Princeton University Press (2011).

[30] C. Caër, S. Combrié, X. Le Roux, E. Cassan, and A. De Rossi, "Extreme optical confinement in a slotted photonic crystal waveguide," Applied Physics Letters 105, p. 121111 (2014).

[31] S.P. Yu, J.D. Hood, J.A. Muniz, M.J. Martin, R. Norte, C.L. Hung, S.M. Meenehan, J.D. Cohen, O. Painter, and H.J. Kimble, "Nanowire photonic crystal waveguides for single-atom trapping and strong light-matter interactions," Applied Physics Letters 104, p. 111103 (2014).

[32] W.L. Barnes, A. Dereux, and T.W. Ebbesen, "Surface plasmon subwavelength optics," Nature 424, p. 824-830 (2003).

[33] D.K. Gramotnev, and S.I. Bozhevolnyi, "Nanofocusing of electromagnetic radiation," Nature Photonics 8, p. 13-22 (2014).

[34] Y. Liu, T. Zentgraf, G. Bartal, and X. Zhang, "Transformational plasmon optics," Nano Letters 10, p. 1991-1997 (2010).

[35] P.A. Huidobro, M.L. Nesterov, L. Martin-Moreno, and F.J. Garcia-Vidal, "Transformation optics for plasmonics," Nano Letters 10, p. 1985-1990 (2010).

[36] M. Kadic, S. Guenneau, S. Enoch, P.A. Huidobro, L. Martin-Moreno, F.J. Garcia-Vidal, J. Renger, and R. Quidant, "Transformation plasmonics," Nanophotonics 1, p. 51-64 (2012).

[37] I.I. Smolyaninov, V.N. Smolyaninova, A.V. Kildishev, and V.M. Shalaev,"Anisotropic metamaterials emulated by tapered waveguides: application to optical cloaking," Physical Review Letters 102, p. 213901 (2009).

[38] A. Vakil and N. Engheta, "Transformation optics using graphene," Science 332, p. 1291-1294 (2011).

[39] B. Dastmalchi, P. Tassin, T. Koschny, and C.M. Soukoulis, "A new perspective on plasmonics: confinement and propagation length of surface plasmons for different materials and geometries," Advanced Optical Materials 4, p. 177-184 (2016). 
[40] J.D. Caldwell, A.V. Kretinin, Y. Chen, V. Giannini, M.M. Fogler, Y. Francescato, C.T. Ellis, J.G. Tischler, C.R. Woods, A.J. Giles, M. Hong, K. Watanabe, T. Taniguchi, S.A. Maier, and K. S. Novoselov, "Subdiffractional volume-confined polaritons in the natural hyperbolic material hexagonal boron nitride," Nature Communications 5, p. 5221 (2014).

[41] P. Li, M. Lewin, A.V. Kretinin, J. Caldwell, K.S. Novoselov, T. Taniguchi, K. Watanabe, F. Gaussmann, and T. Taubner, "Hyperbolic phonon-polaritons in boron nitride for near-field optical imaging and focusing," Nature Communications 6, p. 8507 (2015).

[42] O. Takayama, D. Artigas, and L. Torner, "Lossless directional guiding of light in dielectric nanosheets using Dyakonov surface waves," Nature Nanotechnology 9, p. 419-424 (2014).

[43] S. Jahani and Z. Jacob, “All-dielectric metamaterials," Nature Nanotechnology 11, p. 23-36 (2016).

[44] S.J. Corbitt, M. Francoeur, and B. Raeymaekers, "Implementation of optical dielectric metamaterials: A review," Journal of Quantitative Spectroscopy and Radiative Transfer 158, p. 3-16 (2015).

[45] J. Fischer, T. Ergin, and M. Wegener, "Three-dimensional polarization-independent visible-frequency carpet invisibility cloak," Optics Letters 36, p. 2059-2061 (2011).

[46] U. Zywietz, A.B. Evlyukhin, C. Reinhardt, and B.N. Chichkov, "Laser printing of silicon nanoparticles with resonant optical electric and magnetic responses," Nature Communications 5, p.3402 (2014).

[47] C.B. Arnold, P. Serra, and A. Piqué, "Laser direct-write techniques for printing of complex materials," MRS Bulletin 32, p. 23-31 (2007).

[48] P. Moitra, B.A. Slovick, W. Li, I.I. Kravchencko, D.P. Briggs, S. Krishnamurthy, and J. Valentine, "Large-scale all-dielectric metamaterial perfect reflectors," ACS Photonics 2 , p. 692-698 (2015).

[49] S. Viaene, V. Ginis, J. Danckaert, and P. Tassin, "Transforming two-dimensional guided light using nonmagnetic metamaterial waveguides," Physical Review B 93, p. 085429 (2016).

[50] R. Schinzinger and P.A.A. Laura, [Conformal Mapping: Methods and Applications], Dover Publications, New York, (2003). 